\title{
3-D Vector Velocity Estimation with Row-Column Addressed Arrays
}

Holbek, Simon; Christiansen, Thomas Lehrmann; Rasmussen, Morten Fischer; Stuart, Matthias Bo; Thomsen, Erik Vilain; Jensen, Jørgen Arendt

\section{Published in:}

Proceedings of IEEE International Ultrasonics Symposium

\section{Publication date:}

2015

Document Version

Peer reviewed version

Link back to DTU Orbit

Citation (APA):

Holbek, S., Christiansen, T. L., Rasmussen, M. F., Stuart, M. B., Thomsen, E. V., \& Jensen, J. A. (2015). 3-D Vector Velocity Estimation with Row-Column Addressed Arrays. In Proceedings of IEEE International Ultrasonics Symposium IEEE.

\section{General rights}

Copyright and moral rights for the publications made accessible in the public portal are retained by the authors and/or other copyright owners and it is a condition of accessing publications that users recognise and abide by the legal requirements associated with these rights.

- Users may download and print one copy of any publication from the public portal for the purpose of private study or research.

- You may not further distribute the material or use it for any profit-making activity or commercial gain

- You may freely distribute the URL identifying the publication in the public portal 


\title{
3-D Vector Velocity Estimation with Row-Column Addressed Arrays
}

\author{
Simon Holbek*, Thomas Lehrmann Christiansen ${ }^{\dagger}$, Morten Fischer Rasmussen*, \\ Matthias Bo Stuart*, Erik Vilain Thomasen ${ }^{\dagger}$ and Jørgen Arendt Jensen* \\ * Center for Fast Ultrasound Imaging, Dept. of Elec. Eng., Bldg. 349, Technical University of Denmark, \\ 2800 Kgs. Lyngby, Denmark \\ $\dagger$ Department of Micro- and Nanotechnology, \\ Technical University of Denmark, 2800 Kgs. Lyngby, Denmark
}

\begin{abstract}
The concept of 2-D row-column (RC) addressed arrays for 3-D imaging have shown to be an interesting alternative to 2-D matrix array, due to the reduced channel count. However, the properties for RC arrays to estimate blood velocities have never been reported, which is of great importance for a clinical implementation of this type of array. The aim of this study is, thus, to develop a technique for estimating 3-D vector flow with a RC array using the transverse oscillation (TO) method. The properties are explored both in a simulation study and with a prototype probe for experimental use. In both setups, a 124 channel 2-D RC array with integrated apodization, pitch $=270 \mu \mathrm{m}$ and a center frequency of $3.0 \mathrm{MHz}$ was used. The performance of the estimator was tested on a simulated vessel $(\emptyset=12 \mathrm{~mm})$ with a parabolic flow profile and a peak velocity of $1 \mathrm{~m} / \mathrm{s}$. Measurements were made in a flowrig $(\varnothing=12 \mathrm{~mm})$ containing a laminar parabolic flow and a peak velocity of 0.54 $\mathbf{m} / \mathbf{s}$. Data was sampled and stored on the experimental ultrasound scanner SARUS. Simulations yields relative mean biases at ($1.1 \%,-1.5 \%,-1.0 \%$ ) with mean standard deviations of $\tilde{\sigma}$ were $(8.5 \%, 9.0 \%, 1.4 \%) \%$ for $\left(v_{x}, v_{y}, v_{z}\right)$ from a 3-D velocity vector in a $15^{\circ}$ rotated vessel with a $75^{\circ}$ beam-to-flow angle. In the experimental setup with a $90^{\circ}$ beam-to-flow angle, the relative mean biases were $(-2.6 \%,-1.3 \%, 1.4 \%)$ with a relative standard deviation of $(5.0 \%, 5.2 \%, 1.0 \%)$ for the respective transverse, lateral and axial velocity component.
\end{abstract}

\section{INTRODUCTION}

Blood velocity estimation with ultrasound has become a commonly used tool for diagnosing cardiovascular diseases [1]. However, even though blood propagates in all three directions, commercial scanners are only capable of estimating 1-D or 2-D blood velocities. To obtain the last velocity component for estimating the true blood flow, a 2-D transducer can be used. A 32x32 element 2-D matrix array (Vermon S.A., Tours, France) was previously used to estimate $3-\mathrm{D}$ vector flow in combination with the transverse oscillation method (TO) [2] [3] and a similar probe was used to estimate axial velocities in a volume using Doppler techniques [4]. One major drawback with the matrix probe is that the total number of interconnections in a $N \times N$ element transducer, scales with $N^{2}$, which causes fabrication issues. Thus, solutions for 3-D imaging with a sparse 2-D transducer have been investigated in the literature.

One of the ideas that have emerged, was a to create a 2-D row-column (RC) addressed array [5], [6]. A RC addressed array can be viewed as two orthogonally oriented 1-D arrays mounted on top of each other. In a 2-D RC addressed array the total number of elements is reduced to $N+N$, which allows for 2-D arrays with a large footprint at a sparse channel count. This work develops a method for 3-D vector flow estimation using a 2-D RC addressed array. The applied velocity estimator is a modified 3-D version of the transverse oscillation method [7], [8], [9]. 3-D vector flow estimates are shown for both Field II [10] [11] simulations and an experimental setup using a 2$\mathrm{D}$ RC prototype probe with similar properties and transducer specifications.

\section{2-D ROW-COLUMN ADDRESSED ARRAYS}

The concept of a 2-D RC addressed array is easiest described as a $1 \times N$ and a $N \times 1$ 1-D linear array merged together to form a $N+N$ array which can be accessed from either its row indices or column indices as seen in Fig. 1. In transmit, any number or combination of elements can be excited at the same time as long as they are oriented in the same direction. In receive, however, data from all $2 N$ elements can be accessed simultaneously. Focusing can be performed in one lateral dimension by electronically applying a time delay to each element.



Fig. 1. Two $1 \times N$ and $N \times 1$ 1-D one-way focused linear array are mounted orthogonal on top of each other to form the 2-D row-column addressed $N+N$ array.

To some extent, a 2-D RC array is a special case of a 2-D matrix array, where whole lines of elements are locked together to work as a unit. The reduced flexibility in transmit and the lack of access to element data from $N^{2}$ channels with a $\mathrm{RC}$ array translates to reduced fabrication complexity and data processing requirements. It is a very costly and complicated task to dice out a $N \times N$ piezo ceramic and to provide electrical 
connections to each element, if a large value is chosen for $N$. The amount of data to be processed is also reduced with a factor of $N / 2$ for a $N+N$ RC array with same aperture size as a $N \times N$ matrix array, which eases a real-time implementation.

The drawback of using RC arrays are their long element, which produce several ghost echoes emerging from the element edges [6],[12]. The amplitude of the most powerful ghost echo is approximately $40 \mathrm{~dB}$ lower than the amplitude of the main echo. It was shown that edge waves could be reduced, without affecting the main echo, by implementing a roll-off apodization at the end of each line element [13]. Based on these suggestions, each end of the line elements included a Hann windowed roll-off apodization of length $16 \times$ pitch. Integrated apodization was applied in both the simulations and in the 2-D RC prototype probe used for the experimental setup.

\section{MATERIALS \& METHODS}

The section describes the developed technique for 3-D vector flow estimation using a $2-\mathrm{D} \mathrm{RC}$ array. It introduces the velocity estimator, the emission seqeunce, the applied RC array and the simulation and measurement environment. The describtion of the method was applied in both cases unless otherwise stated.

\section{A. The transverse oscillation method}

The TO estimator is a method for estimating 2-D vector flow by generating two decoupled double-oscillating fields in receive. The received signals are affected by axial or transverse blood flow motion which propagates through the doubleoscillating fields. By using a phase-shift estimator, the axial and transverse motion can be detected. For a more thorough explanation of the theory, please consider [7], [14], [2] for further reading.

In general, three beamformed lines are needed for estimating 2-D vector flow with TO. One is needed as input for the conventional axial estimator [15] and two lines are needed for the transverse velocity component. The two lines used for transverse estimates are beamformed along the directions $\left( \pm \lambda_{x}(z), 0, z\right)$, where $\lambda_{x}(z)$ is given by

$$
\lambda_{x}(z)=2 \lambda_{z} \frac{z}{d_{x}}
$$

where $\lambda_{z}$ is the wavelength of the emitted pulse in the medium. The wavelength is controllable and determined by the receive apodization profile. The apodization should contain two symmetrically placed peaks separated by a distance $d_{x}$. At a certain axial depth $z, \lambda_{x}$. The maximum transverse velocity one can estimate without reaching the aliasing limis is then

$$
v_{x_{\max }}=\frac{\lambda_{x}}{4 k} f_{p r f},
$$

where $k$ is the lag used in the autocorrelation and $f_{p r f}$ is the pulse repetition frequency between two similar emissions. To estimate the third velocity component $v_{y}$ two additional lines have to be beamformed along the directions $\left(0, \pm \lambda_{y}(z), z\right)$. A total of five beamformed lines are therefore required to estimate $3-\mathrm{D}$ vector flow with the TO method.

\section{B. Emissons sequence}

A total of 5 beamformed lines are needed to estimate 3-D vector flow along a line with TO. But since it is not possible to generate a focal point with a RC array, from which all 5 lines could be beamformed simultaneously an interleaved sequence was designed. The principle is illustrated in Fig. 2. One focused transmission (transmit 1) is made on the row elements and received on column elements (receive 1). From this, three of the five needed lines can be beamformed and the two velocity components $v_{z}$ and $v_{y}$ along the centerline can be derived. In the opposite scenario with transmit on the columns (transmit 2) and receive on the rows (receive 2), the $v_{z}$ and $v_{x}$ velocity components can be found. The applied emissions sequence is, thus, made by first transmitting on the row and receiving on the columns and afterwards emit with columns and receive on the rows. By combining the two transverse estimates found from each transmit event with an axial estimate, the 3-D vector velocities are found. The described sequence was repeated 32 times for each frame. A total of 100 frames, each containing $2 \times 32$ emissions was obtained in the simulation study and 20 frames were acquired in the experiments. The focal line were in both cases at $3 \mathrm{~cm}$ depth.

\section{The 2-D RC array}

A $62+62$ element 2-D RC array was used in this study. The simulated RC array and the prototype $\mathrm{RC}$ probe had same properties, resulting in a pitch $=0.27 \mathrm{~mm}$, kerf $=0.025 \mathrm{~mm}$, sampling frequency $=70 \mathrm{MHz}$, center frequency $f_{0}=3.0 \mathrm{MHz}$ and a footprint length in each dimension of $1.73 \mathrm{~cm}$, see Table I. A 6 cycle sinusoidal pulse was used in transmit.



Fig. 2. Illustration of the transmit and receive scenario. First, focused ultrasound is emitted on the rows (transmit 1) and receive on the columns (receive 1). Secondly, the scenario is flipped, and the focused ultrasound is emitted on the columns (transmit 2) and received on the rows (receive 2). The axial velocity can be estimated in both cases. But with the TO approach only the velocity component perpendicular to the receive elements can be estimated. Gray elements are active ones, whereas white elements are inactive according to the required TO apodization profiles (conceptually illustrated by the gray areas).

\section{Simulation Setup}

A 20x20x20 mm cubic phantom containing a cylindrical blood vessel $(\varnothing=12 \mathrm{~mm})$ located at $3 \mathrm{~cm}$ depth was defined for the simulations. Scatterers inside the cylinder were translated according to a circular symmetric parabolic velocity profile, and scatters outside the cylinder were considered 


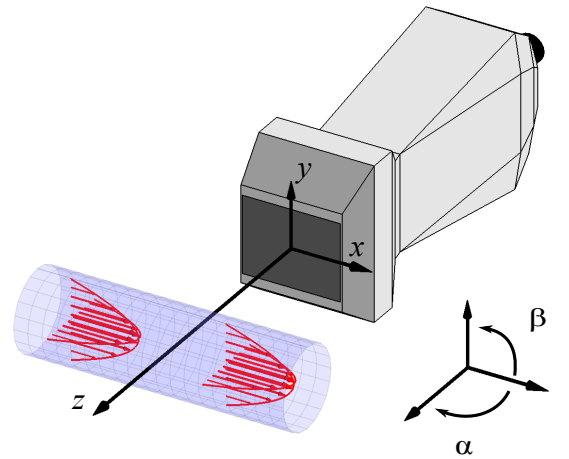

Fig. 3. illustration of the simulation setup and the two rotation angles $\alpha$ and $\beta$, where a row-columns transducer array is located $3 \mathrm{~cm}$ from the center of the emulated blood vessel.

stationary. The peak velocity $v_{0}$ in the parabolic flow was $1 \mathrm{~m} / \mathrm{s}$ and propagating in the $x$-direction as default. Rotation of the scatter phantom could be made around two axes; around the $y$-axis, denoted the beam-to-flow angle $\alpha$ and rotation around the $z$-axis denoted $\beta$. An illustration of the setup is seen in Fig. 3. Different combinations of $\alpha$ and $\beta$ were investigated and the $f_{p r f}$ was set to [3000 50009700 13800] $\mathrm{Hz}$ for $\alpha=\left[90^{\circ} 75^{\circ} 60^{\circ} 45^{\circ}\right]$ respectively, to ensure that the aliasing limit was not exceeded for any of the three velocity components.

\section{E. Experimental Setup}

The 2-D RC prototype probe was connected to the experimental ultrasound scanner, SARUS [16] from which raw RF data was sampled for all active channels. The probe was fixated above a rubber tube $(\varnothing=12 \mathrm{~mm})$ submerged in water at a distance of $3 \mathrm{~cm}$ from the center of the vessel. An illustration of the setup is seen in Fig. 3. The beamto-flow angle $\alpha$ was $90^{\circ}$ and $\beta=0^{\circ}$. A centrifugal pump circulated a blood-mimicking fluid (Danish Phantom Design, Frederikssund, Denmark) which ensured a fully developed laminar flow with a parabolic profile. The peak velocity was estimated to be $0.54 \mathrm{~m} / \mathrm{s}$ based on the vessel diameter and the flow rate measured with a magnetic MAG1100 (Danfoss, Nordborg, Denmark) flowmeter. A lower velocity magnitude, compared to the simulation setup was chosen in the flow rig to avoid intake of air bubbles in the system. The $f_{p r f}$ was was therefore scaled accordingly to $1.4 \mathrm{kHz}$ to match the expected velocity range.

\section{DATA PROCESSING}

The stored data was processed offline. Due to the one-way focusing with a 2-D RC array, only sampled data from row elements were processed, when column elements were used as transmitters and vice versa, as illustrated in Fig. 2. The raw RF data was match filtered and beamformed with a dedicated RC beamformer [13]. For each transmit event, one line was beamformed for the axial velocity estimator and two separated lines were beamformed and used for estimating the velocity component orthogonal to the receiving elements. For the axial estimates, a Hann apodization was applied in receive, and for the transverse estimates two separated Hann peaks as sketched
TABLE I. Transducer And Emissions Sequence SetuP

\begin{tabular}{ll}
\hline \multicolumn{2}{c}{ Transducer } \\
Parameter & Value \\
\hline Trandsucer type & 2 -D Row-Column \\
No. of elements in x & 62 \\
No. of elements in y & 62 \\
Pitch in x & $0.27 \mathrm{~mm}$ \\
Pitch in y & $0.27 \mathrm{~mm}$ \\
Height & $0.245 \mathrm{~mm}$ \\
Kerf & $0.025 \mathrm{~mm}$ \\
Sampling frequency & $70 \mathrm{MHz}$ \\
Center frequency & $3.0 \mathrm{MHz}$ \\
Footprint size & $1.73 \mathrm{~cm}$ \\
\hline
\end{tabular}

in Fig. 2 were used. The two TO apodization peaks were spaced a distance of $35 \times$ pitch and had a width of $25 \times$ pitch. An autocorrelation approach was used for the axial velocity estimate [15] and the transverse oscillation (TO) method [7][8] was applied for velocity estimates in the transverse and elevation direction. A more extensive description of the 3-D TO method is described in previous works [2], [3].

\section{Results}

The simulated flow phantom was rotated in different angle combinations of $\alpha$ and $\beta$. A total of 100 frames were simulated for each constellation and processed. The ability to estimate angle-independent peak velocities from the 3 -D velocity vector is shown in Fig. 4. For a $90^{\circ}$ beam-to-flow, the estimated peak velocity is found to be within $1.3-1.6 \%$ of $v_{0}$ for any investigated $\beta$ rotation. Decreasing $\alpha$ in general leads to a higher standard deviation, which increases from a mean value of $4.6 \%$ for $\alpha=90^{\circ}$, to $5.8 \%, 8.2 \%$ and $15.2 \%$ for $\alpha=75^{\circ}$ , $60^{\circ}$ and $45^{\circ}$ respectively.

A representative selection of three estimated velocity components is seen in Fig. 5. The three velocity components were estimated from a simulated setup where the vessel was rotated in a beam-to-flow angle $\alpha=75^{\circ}$ and $\beta=15^{\circ}$. The mean relative bias $\tilde{B}$ was $(-1.1,-1.5,-1.0) \%$ for the velocity components $\left(v_{x}, v_{y}, v_{z}\right)$. The corresponding mean standard deviation $\tilde{\sigma}$ were $(8.5,9.0,1.4) \%$. The peak velocity was $0.97 \mathrm{~m} / \mathrm{s}$ compared to the expected $1 \mathrm{~m} / \mathrm{s}$.

A total of 20 frames were recorded for the experimental setup and processed offline. The beam-to-flow angle $\alpha=90^{\circ}$ and $\beta=0^{\circ}$. The experimental results gave $\tilde{B}=(-2.6,-1.3,1.4)$ $\%$ for the velocity components $\left(v_{x}, v_{y}, v_{z}\right)$ with $\tilde{\sigma}=(5.0,5.2$, 1.0) $\%$, see Fig. 6.

\section{CONCLUSION}

The presented results demonstrate that a developed technique for 3-D vector flow estimation could be implemented

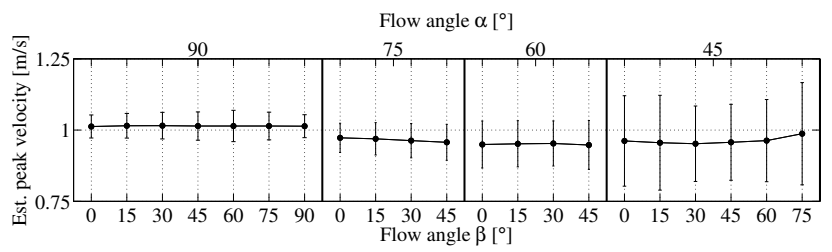

Fig. 4. Peak velocities derived from the estimated 3-D vector velocities as a function of vessel orientation. 


\section{Simulated 3-D Vector Flow}
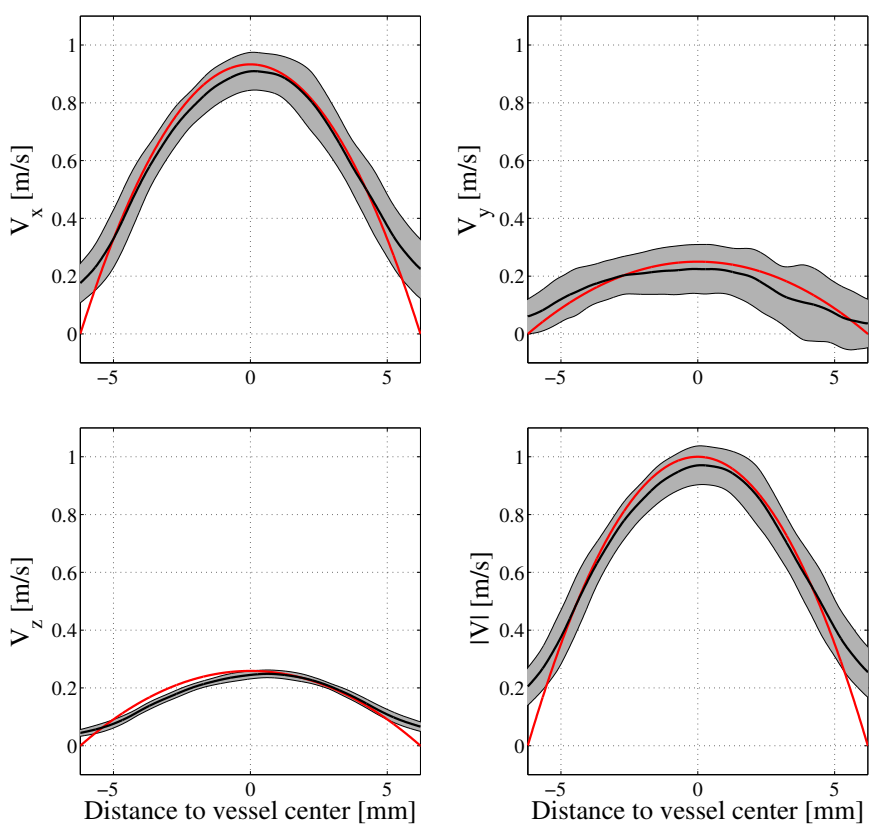

Fig. 5. Simulated 3-D vector flow estimation decomposed into the three velocity components and the absolute velocities. The red line indicates the theoretical velocity profile along with the estimated profile (black) and \pm one standard deviation (gray area).

\section{Measured 3-D Vector Flow}


Fig. 6. Measured 3-D vector flow estimation decomposed into the three velocity components and the absolute velocities. The red line indicates the theoretical velocity profile along with the estimated profile (black) and \pm one standard deviation (gray area).

on a 2-D RC addressed arrays using the TO method. The simulated results were in good agreement with the expected velocities. Although slightly underestimating the velocities in the experimental setup, the expected parabolic velocity profile could be estimated. However, the results are very promissing and demonstrates the potential of $\mathrm{RC}$ addressed arrays for velocity estimation.

\section{ACKNOWLEDGMENT}

This work was supported by grant 82-2012-4 from the Danish National Advanced Technology Foundation and by BK Ultrasound Aps.

\section{REFERENCES}

[1] G. M. von Reutern, M. W. Goertler, N. M. Bornstein, M. D. Sette, D. H. Evans, A. Hetzel, M. Kaps, F. Perren, A. Razumovky, and et al., "Grading carotid stenosis using ultrasonic methods," Stroke, vol. 43, no. 3, pp. 916-921, 2012.

[2] M. J. Pihl and J. A. Jensen, "A transverse oscillation approach for estimation of three-dimensional velocity vectors. Part I: Concept and simulation study," IEEE Trans. Ultrason., Ferroelec., Freq. Contr., vol. 61, pp. 1599-1607, 2014.

[3] M. J. Pihl, M. B. Stuart, B. G. Tomov, M. F. Rasmussen, and J. A. Jensen, "A transverse oscillation approach for estimation of threedimensional velocity vectors. Part II: Experimental validation," IEEE Trans. Ultrason., Ferroelec., Freq. Contr., vol. 51, no. 10, pp. 16081618, 2014.

[4] J. Provost, C. Papadacci, J. E. Arango, M. Imbault, M. Fink, J. L. Gennisson, M. Tanter, and M. Pernot, "3-D ultrafast ultrasound imaging in vivo," Physics in Medicine and Biology, vol. 59, no. 19, pp. L1-L13, 2014.

[5] C. E. Morton and G. R. Lockwood, "Theoretical assessment of a crossed electrode 2-D array for 3-D imaging," in Proc. IEEE Ultrason. Symp., 2003, pp. 968-971.

[6] C. E. M. Démoré, A. Joyce, K. Wall, and G. Lockwood, "Real-time volume imaging using a crossed electrode array," IEEE Trans. Ultrason., Ferroelec., Freq. Contr., vol. 56, no. 6, pp. 1252-1261, 2009.

[7] J. A. Jensen and P. Munk, "A new method for estimation of velocity vectors," IEEE Trans. Ultrason., Ferroelec., Freq. Contr., vol. 45, pp. 837-851, 1998.

[8] J. A. Jensen, "A new estimator for vector velocity estimation," IEEE Trans. Ultrason., Ferroelec., Freq. Contr., vol. 48, no. 4, pp. 886-894, 2001 .

[9] T. L. Christiansen, S. Holbek, M. F. Rasmusen, E. V. Thomasen, and J. A. Jensen, "3-D flow estimation using row-column addressed transducer arrays," US application number: 14/599,857, January 2015.

[10] J. A. Jensen, "Field: A program for simulating ultrasound systems," Med. Biol. Eng. Comp., vol. 10th Nordic-Baltic Conference on Biomedical Imaging, Vol. 4, Supplement 1, Part 1, pp. 351-353, 1996.

[11] J. A. Jensen and N. B. Svendsen, "Calculation of pressure fields from arbitrarily shaped, apodized, and excited ultrasound transducers," IEEE Trans. Ultrason., Ferroelec., Freq. Contr., vol. 39, pp. 262-267, 1992.

[12] M. F. Rasmussen and J. A. Jensen, "3D ultrasound imaging performance of a row-column addressed 2D array transducer: a simulation study," in Proc. SPIE Med. Imag., 2013, pp. 1-11, 86750C.

[13] M. F. Rasmussen, T. L. Christiansen, E. V. Thomsen, and J. A. Jensen, "3-D imaging using row-column-addressed arrays with integrated apodization - Part I: Apodization design and line element beamforming," IEEE Trans. Ultrason., Ferroelec., Freq. Contr., vol. 62, no. 5, pp. 947-958, 2015.

[14] J. Udesen and J. A. Jensen, "Investigation of Transverse Oscillation Method," IEEE Trans. Ultrason., Ferroelec., Freq. Contr., vol. 53, pp. 959-971, 2006.

[15] C. Kasai, K. Namekawa, A. Koyano, and R. Omoto, "Real-Time TwoDimensional Blood Flow Imaging using an Autocorrelation Technique," IEEE Trans. Son. Ultrason., vol. 32, pp. 458-463, 1985.

[16] J. A. Jensen, H. Holten-Lund, R. T. Nilsson, M. Hansen, U. D. Larsen, R. P. Domsten, B. G. Tomov, M. B. Stuart, S. I. Nikolov, M. J. Pihl, Y. Du, J. H. Rasmussen, and M. F. Rasmussen, "SARUS: A synthetic aperture real-time ultrasound system," IEEE Trans. Ultrason., Ferroelec., Freq. Contr., vol. 60, no. 9, pp. 1838-1852, 2013. 